\title{
DNA methylation in the human frontal cortex reveals a putative mechanism for age-by-disease interactions
}

\author{
Brandon C. McKinney (1)', Chien-Wei Lin², Tanbin Rahman², Hyunjung Oh'1,3, David A. Lewis (1)', George Tseng ${ }^{2}$ and
} Etienne Sibille ${ }^{1,3}$

\begin{abstract}
A consistent gene set undergoes age-associated expression changes in the human cerebral cortex, and our Age-byDisease Model posits that these changes contribute to psychiatric diseases by "pushing" the expression of diseaseassociated genes in disease-promoting directions. DNA methylation (DNAm) is an attractive candidate mechanism for age-associated gene expression changes. We used the Illumina HumanMethylation450 array to characterize genomewide DNAm in the postmortem orbital frontal cortex from 20 younger ( $<42$ years) and 19 older ( $>60$ years) subjects. DNAm data were integrated with existing normal brain aging expression data and sets of psychiatric disease risk genes to test the hypothesis that age-associated DNAm changes contribute to age-associated gene expression changes and, by extension, susceptibility to psychiatric diseases. We found that age-associated differentially methylated regions (aDMRs) are common, robust, bidirectional, concentrated in CpG island shelves and sea, depleted in CpG islands, and enriched among genes undergoing age-associated expression changes $\left(\mathrm{OR}=2.30, p=1.69 \times 10^{-27}\right)$. We found the aDMRs are enriched among genetic association-based risk genes for schizophrenia, Alzheimer's disease (AD), and major depressive disorder (MDD) $(\mathrm{OR}=2.51, p=0.00015 ; \mathrm{OR}=2.38, p=0.036$; and $\mathrm{OR}=3.08, p=0.018$, respectively) as well as expression-based MDD-associated genes $(O R=1.48, p=0.00012)$. Similar patterns of enrichment were found for aDMRs that correlate with local gene expression. These results were replicated in a large publically-available dataset, and confirmed by meta-analysis of the two datasets. Our findings suggest DNAm is a molecular mechanism for age-associated gene expression changes and support a role for DNAm in age-by-disease interactions through preferential targeting of disease-associated genes.
\end{abstract}

\section{Introduction}

Ameliorating the burden of psychiatric diseases, including schizophrenia (SZ), Alzheimer's Disease (AD), and major depressive disorder $(\mathrm{MDD})^{1}$ will require advances in their prevention and treatment, and such advances require a neurobiological understanding of their etiology and pathophysiology. Previously, we proposed the

\footnotetext{
Correspondence: Brandon C. McKinney (mckinneybc@upmc.edu) or Etienne Sibille (Etienne.sibille@camh.ca)

'Department of Psychiatry, University of Pittsburgh, Pittsburgh, PA, USA

${ }^{2}$ Department of Biostatistics, University of Pittsburgh, Pittsburgh, PA, USA

Full list of author information is available at the end of the article.

These authors contributed equally: Brandon C. McKinney, Chien-Wei Lin
}

Age-by-Disease Model, a neurobiological model for psychiatric diseases $^{2-4}$. This model posits that many psychiatric diseases are, in part, the result of anticipated age-associated changes in the expression of diseaseassociated genes, and that individual variability in rates of age-dependent changes determines risk or resiliency to develop age-related disorders ${ }^{2-4}$.

The upstream mechanisms giving rise to age-associated gene expression changes are incompletely understood. Candidate mechanisms include loss of telomere integrity, increased oxidative stress, and epigenetic modifications (reviewed in the ref. ${ }^{2}$ ). DNA methylation (DNAm), the covalent addition of a methyl group to a cytosine 
nucleotide in DNA, is an epigenetic modification. Two characteristics of DNAm make it a particularly attractive candidate mechanism. Firstly, changes in DNAm occur throughout the lifespan ${ }^{5}$. DNAm tends to increase with age near genes and decrease with age in intergenic regions ${ }^{6}$. Locus-specific DNAm variability tends to increase with age and is thought to result from each individual's exposure to a unique set of environmental factors $^{7-9}$. Secondly, changes in DNAm near gene regulatory regions affect local gene expression. Generally, gene expression is negatively correlated with DNAm near the transcriptional start site of a gene, and positively correlated with intragenic DNAm ${ }^{10}$.

In this study, we tested the hypothesis that ageassociated DNAm changes contribute to age-associated gene expression changes and, by extension, susceptibility to psychiatric diseases. We predicted that genes that undergo age-associated gene expression changes in the brain would be enriched in differentially methylated regions (DMRs). We also predicted that genes associated with risk for $\mathrm{AD}$, a psychiatric disease associated with advanced age, would be enriched in age-associated DMRs (aDMRs) whereas genes associated with risk for SZ, a psychiatric disease associated with neurodevelopment, would not be enriched in aDMRs. Further, we predicted MDD, a psychiatric disease that can present for the first time or recur at any adult age, would exhibit results intermediate between those for AD and SZ.

\section{Methods}

\section{Postmortem brains}

Brains were recovered during autopsies conducted at the Allegheny County Medical Examiner's Office, Pittsburgh, PA, following informed consent from the next-ofkin. DSM-IV diagnoses, including substance use disorders, or absence thereof, were determined by clinicians using medical records, structured interviews with surviving relatives, and toxicology reports. The right hemisphere of each brain was cut coronally, immediately frozen, and stored at $-80^{\circ} \mathrm{C}$. Samples containing all six cortical layers, but excluding adjacent white matter, were harvested from cryostat sections of the orbital frontal cortex (OFC), specifically Brodmann Areas 11 and 47 . Procedures were approved by the University of Pittsburgh Committee for the Oversight of Research and Clinical Trials Involving the Dead and the Institutional Review Board for Biomedical Research.

\section{Cohort membership}

The younger and older groups comprised 22 subjects $\leq 42$ years of age and 22 subjects $\geq 60$ years of age, respectively, without DSM-IV diagnoses or neurologic disease. Age cut-offs were determined by considering availability of subjects with reasonable matching of
Table 1 Group characteristics. Data for continuous variables are presented as group average \pm SEM

\begin{tabular}{lll}
\hline Group & Younger & Older \\
\hline Number & 20 & 19 \\
Sex & $15 \mathrm{M}, 5 \mathrm{~F}$ & $15 \mathrm{M}, 4 \mathrm{~F}$ \\
Race & $20 \mathrm{~W}$ & $19 \mathrm{~W}$ \\
Age (years)* & $29.75 \pm 2.04$ & $69.32 \pm 2.05$ \\
PMI (hours) & $17.00 \pm 1.47$ & $17.58 \pm 1.61$ \\
Brain pH* & $6.65 \pm 0.05$ & $6.78 \pm 0.05$ \\
RIN & $8.22 \pm 0.14$ & $8.07 \pm 0.15$ \\
BDNF expression & $24.91 \pm 0.68$ & $20.06 \pm 0.79$ \\
SST expression ${ }^{\mathrm{a}^{*}}$ & $675.37 \pm 24.67$ & $385.97 \pm 38.34$ \\
\hline
\end{tabular}

$F$ female, $M$ male, $P M I$ postmortem interval, RIN RNA integrity number, $W$ white, $B$ black

*Group averages are significantly different $(p<0.05)$

${ }^{\mathrm{a}}$ Microarray signal intensities

cofactors, the fact that brain aging is continuous and relatively homogeneous across adult life ${ }^{11}$, and expression levels of biomarkers of brain aging ${ }^{12,13}$. Subjects with the highest (younger group) or lowest (older group) BDNF and SST mRNA levels in our previous study were selected $^{14}$. Because DNAm is highly dependent on race ${ }^{15-18}$ and only 5 subjects were black, they were removed from the cohort, leaving 20 younger, and 19 older, subjects. Groups did not differ with respect to postmortem interval (PMI), RNA integrity number (Agilent, Santa Clara, California, USA), or sex (Table 1 and Supplemental Table 1). Brain $\mathrm{pH}$ was slightly higher in the older group but the significance of such a difference is unclear. All data was collected by a researcher blind to age group.

\section{DNA preparation and bisulfite conversion}

DNA was isolated from OFC gray matter using AllPrep DNA/RNA/Protein Mini Kit (Qiagen, Valencia, CA, USA) and bisulfite-converted using EZ-96 DNA Methylation Kit (Zymo Research, Irvine, CA, USA), both as per manufacturer's protocol.

\section{DNAm arrays}

DNAm is the addition of a methyl group to a cytosine nucleotide within the context of a cytosine-phosphateguanine $(\mathrm{CpG})$ dinucleotide, usually, but also within the context of a cytosine-phosphate- $\mathrm{H}$ dinucleotide $(\mathrm{CpH} ; \mathrm{H}$ $=$ adenine, cytosine, or thymine), sometimes ${ }^{19}$. CpGs and $\mathrm{CpHs}$ are referred to as "DNAm sites" or "sites" in this manuscript. DNAm was measured at 485,577 sites (482,421 CpG dinucleotides, $3091 \mathrm{CpH}$ dinucleotides, and 65 SNPs) using HumanMethylation450 array (HM450 array; Illumina, San Diego, CA, USA) as per manufacturer's protocol. The DNAm level at a site was 
expressed as a $\beta$-value, the ratio of signal from a methylated probe relative to the sum of both methylated and unmethylated probes.

In an earlier study of somatostatin in the OFC, which included a subset of the same subjects assessed in the present study, a strong correlation was observed between DNAm levels measured by DNAm array and those measured by pyrosequencing ${ }^{16}$.

\section{Data preprocessing and filtering}

Analyses of the data were performed using the R software environment (www.r-project.org).

Data preprocessing (color adjustment, background correction, and quantile normalization) was performed using Bioconductor lumi. Data from poorly hybridizing probes, probes on the $\mathrm{X} \& \mathrm{Y}$ chromosomes, probes with SNPs in the probe or target site, and probes that map to multiple genetic loci were filtered from the dataset. Data from probes corresponding to 317,349 sites remained for analysis $^{20}$ (Supplemental Fig. 1).

\section{Defining candidate regions and differentially methylated regions}

$\beta$-values for each subject were smoothed with a 500base-pair sliding window using Bioconductor methyAnalysis, and then transformed into $M$-values for the purpose of normality approximation. Sites at which DNAm levels differed between groups by two-tailed $t$-test of the $M$-values $(p<0.05)$ were merged into candidate regions (CRs). A CR was defined as a cluster of these sites for which: (a) two consecutive sites were not separated by more than 1000 basepairs and (b) all sites within a given $\mathrm{CR}$ were concordant for direction of effect. The rest of sites were treated as isolated CRs. The 317,349 sites were grouped into 267,249 CRs.

The $p$-value for each CR was derived by combining the $p$-values from all sites within the CR using Fisher's metaanalysis method (FMM). Further, permutation analysis was used. Subject labels were randomly permuted (500 times) and, in each subject permutation, CRs were redefined and $p$-values calculated using FMM. The permuted $p$-value for the ith $\mathrm{CR}$ was then calculated as:

$$
p_{i}^{*}=\frac{\sum_{b=1}^{500} \sum_{j=1}^{m_{b}} I\left(p_{j}^{(b)} \leq p_{i}\right)}{\sum_{j=1}^{500} m_{b}},
$$

where $p_{i}$ is the meta-analyzed $\mathrm{p}$-value of the observed $\mathrm{i}$-th $\mathrm{CR}, p_{j}^{(b)}$ is the meta-analyzed $\mathrm{p}$-value of the observed $\mathrm{j}$-th $\mathrm{CR}$ based on the b-th permutation sample, and $\mathrm{m}_{\mathrm{b}}$ is the total number of CRs based on b-th permutation sample. False discovery rate (FDR) using Benjamini-Hochberg procedure was used to account for multiple testing comparisons. CR-based effect size was meta-analyzed by fixed effect model (FEM) using ß-values. Correlation with expression level for each CR was estimated using the site within the CR that correlated maximally.

A differentially methylated region (DMR) was defined as a CR for which (a) the $q$-value was less than 0.05 and (b) the CR-based effect size was greater than $3 \%$.

\section{Cell population estimation}

Neuron-to-glia proportion in each subject was estimated using a model based on DNAm values from many cell epigenotype specific sites $^{21}$, and it did not differ between groups (Supplemental Figure 4)."

\section{Gene expression}

Gene expression from the OFC of the 39 subjects studied here was previously measured using GeneChip Human Gene 1.1. ST (Affymetrix, Santa Clara, CA, USA), and expression-age associations for each gene were determined by a random intercept model ${ }^{14,22}$. These data were used for enrichment analysis and calculating DNAm-gene expression correlation, and are available for download from Gene Expression Omnibus (GEO; GSE71620).

\section{Enrichment analysis}

Sets of genes that undergo age-associated changes in gene expression ${ }^{14}$ and psychiatric disease risk genes were evaluated for enrichment in age-associated DNAm changes using Fisher's Exact Tests.

\section{Pathway and gene ontology analysis}

Ingenuity Pathway Analysis (QIAGEN, Redwood City, CA, USA) was used to identify canonical pathways and gene ontologies (molecular function) enriched in genes associated with both age-associated DNAm and expression changes as well as those associated with ageassociated DNAm only. $p$-values were ascertained using right-tailed Fisher's exact tests.

\section{Replication dataset}

Data generated by Jaffe and colleagues ${ }^{23}$ from postmortem dorsolateral prefrontal cortex using HM450K arrays were used for replication analysis. Normalized $\beta$-values from 133 subjects without DSM-IV diagnoses or neurologic disease, including 102 subjects $\leq 42$ years of age and 31 subjects $\geq 60$ years of age, were downloaded from GEO (GSE74193), and analyzed exactly as described for the primary dataset.

\section{Meta-analysis of the primary and replication dataset}

For each DNAm site, fixed-effect model was used to summarize the effect sizes and $p$-values from the two 
datasets. The resulting site-based meta-analyzed effect sizes and $p$-values were then used to meta-define CRs following the procedures described for the primary dataset. Region-based effect sizes and correlation were calculated based on meta-defined CRs.

\section{Results}

Age-associated changes in DNAm are enriched in CpG island shelves and sea, and depleted in CpG islands

We classified DNAm sites within age-associated DMRs (aDMR-associated sites; 12,427 ; Fig. 1 ) as belonging to one of the following mutually-exclusive genomic regions: $C p G$ islands are genomic regions $\geq 200$ basepairs in length with GC content $\geq 50 \%$ and a ratio of observed to expected CpG content $\geq 0.6^{24}$; Cp $G$ island shores are the 2 kilobasepairs flanking outward from CpG islands; $C p G$ island shelves are the 2 kilobasepairs flanking outward from CpG island shores; and $C p G$ island sea refers to all remaining genomic regions ${ }^{25}$.

We found that aDMR-associated sites were not distributed to these genomic regions as expected by chance $\left(p=1.2 \times 10^{-296}\right)$. DNAm sites located in the North and South CpG island shelves $(\mathrm{OR}=1.16, p=0.00028$ and $\mathrm{OR}=1.12, p=0.0094$, respectively), and CpG island sea $\left(\mathrm{OR}=1.76, p=2.3 \times 10^{-201}\right)$, are more likely to be associated with an aDMR. In contrast, sites located in CpG islands are less likely $\left(\mathrm{OR}=0.48, p=1.2 \times 10^{-260}\right)$ to be associated with an aDMR (Fig. 2a, b).

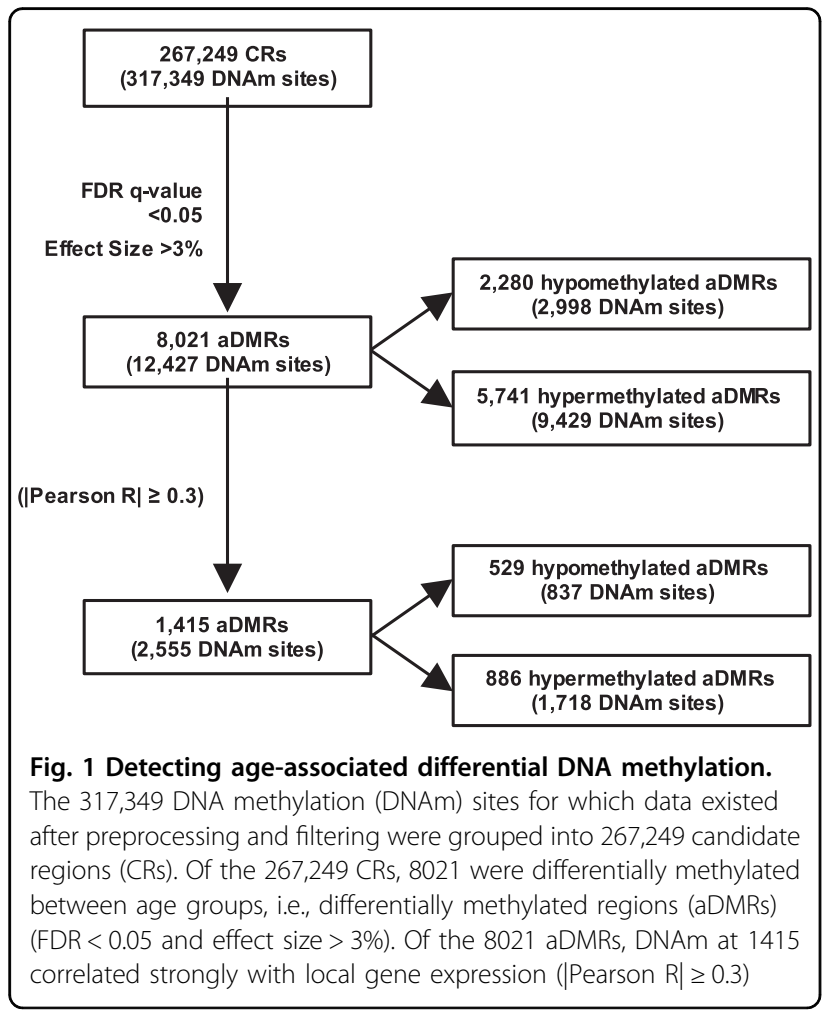

\section{Age-associated hypermethylation is more common in CpG islands}

We found that relative hypermethylation in the older group is more common, in general, than relative hypomethylation. Specifically, $71.6 \%$ of aDMRs and $75.9 \%$ of aDMR-associated sites, are relatively hypermethylated in the older group (Fig. 1). Further, we found that most aDMR-associated sites within CpG islands are relatively hypermethylated in the older group (2276 of 2399 sites, or 94.9\%) and that the percentage of relatively hypermethylated aDMR-associated sites becomes smaller as distance from a CpG island increases (Chi-square $=847.6, \mathrm{df}=5$, $p<2.2 \times 10^{-16}$; Fig. $2 \mathrm{c}$ ) .

\section{Genes that undergo age-associated changes in expression are enriched in aDMRs}

To test if age-associated DNAm changes were preferentially localized to those genes that exhibit ageassociated expression changes, we studied those genes in which expression correlated with age in our previous study ${ }^{14}$. The top genes that undergo age-associated changes in gene expression (top AGE genes) were defined as the $\sim 1000$ genes with expression that correlated most strongly with age (corresponding to $q$-value $\leq$ $9.91 \times 10^{-5}$ in BA47 and $9.58 \times 10^{-5}$ in BA11), whereas the top non-AGE genes were defined as the $\sim 1000$ genes with expression that correlated most weakly with age (corresponding to $q$-value $\geq 0.43$ in BA47 and BA11).

We found that the top AGE genes, but not top nonAGE genes, are enriched in aDMRs (OR $=2.30, p=$ $1.69 \times 10^{-27} ; \mathrm{OR}=0.93, p=0.79$; respectively). Top AGE genes are enriched in aDMRs that are relatively hypomethylated $\left(\mathrm{OR}=2.08, p=3.85 \times 10^{-11}\right)$, and relatively hypermethylated $\left(\mathrm{OR}=2.10, p=7.21 \times 10^{-18}\right)$, with age (Fig. 2d, Supplemental Table 2). Notably, the direction of age-associated changes in DNAm and gene expression are inversely related for genes at which age-associated changes in DNA methylation and gene expression converge (Supplemental Fig. 3).

To increase the likelihood of identifying DNAm sites with particular relevance to age-associated gene expression changes, we integrated DNAm and gene expression data. Of the 8021 aDMRs identified, 1415 were found to correlate with local gene expression with a strength of $\mid$ Pearson's $R \mid \geq 0.3$ (Fig. 1); we henceforth refer to them as expression-correlating aDMRs. Genes that undergo ageassociated expression changes are highly enriched in expression-correlating aDMRs $(\mathrm{OR}=8.58, p=8.76 \times$ $\left.10^{-119}\right)$. To gain insight into the potential biological significance of age-associated changes in DNAm, we performed pathway and gene ontology analysis on genes that both undergo age-associated expression changes and have expression-correlating aDMRs annotated to them $(N=$ 260; Supplemental Table 3). Further, we performed 


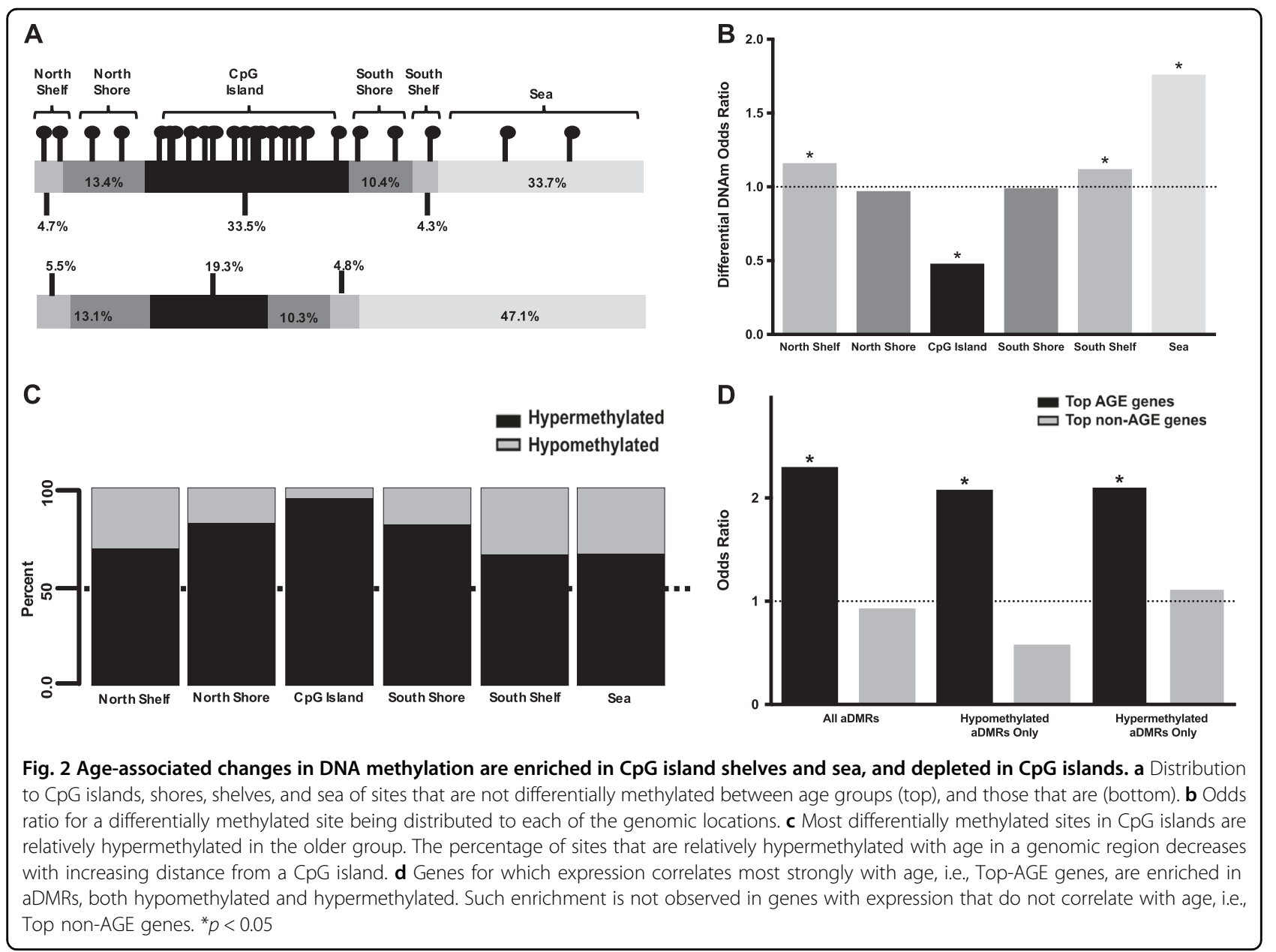

pathway and gene ontology analysis on only genes to which expression-correlating aDMRs were annotated $(N=936)$ (Supplemental Table 6). Both gene sets were enriched $(p<0.05)$ in distinct canonical pathways and gene ontologies related to neuronal signaling (Supplemental Tables 4, 5, 7 \& 8).

Risk genes for psychiatric diseases are enriched in aDMRs

We sought to determine if risk genes for three psychiatric diseases-SZ, AD, and MDD-were enriched for aDMRs. For SZ, risk genes were defined based on closest proximity to one of the top 108 genome-wide association studies (GWAS)-associated common variants ${ }^{26}$. For AD, 27 genes were defined as risk genes based on closest proximity to one of the GWAS-associated common variants $^{27,28}$, or being one of the genes associated with rare deterministic mutations $^{29}$. For MDD, risk genes were defined based on closest proximity to one of the common variants associated with MDD at $p<10^{-5}$ in a recent large GWAS $^{30}$. DNAm data for 78 SZ, 19 MDD, and 25 AD risk genes were available for analysis (Supplemental Table 9).
$\mathrm{SZ}$ risk genes are enriched in aDMRs $(\mathrm{OR}=2.51, p=$ 0.00015 ) (Fig. 3a) and expression-correlating aDMRs (OR $=2.44, p=0.0013$ ) (Fig. 3b). Of GWAS-associated common variants for SZ, 43 are within a protein-coding gene and DNAm data for 40 were available for analysis (Supplemental Table 10 ${ }^{26}$. Enrichment for aDMRs and expression-correlating aDMRs is more robust $(\mathrm{OR}=5.19$, $p=4.7 \times 10^{-7}$ and $\mathrm{OR}=5.53, p=6.3 \times 10^{-5}$; respectively) in this gene subset. AD risk genes are enriched in aDMRs (OR $=2.38, p=0.04)$ (Fig. 3a) and expressioncorrelating aDMRs with marginal significance but high odds ratio (OR $=3.15, p=0.05$ ) (Fig. 3b). Similarly, MDD risk genes are enriched in aDMRs $(\mathrm{OR}=3.08, p=0.02)$ (Fig. 3a) and expression-correlating aDMRs with trendlike significance but high odds ratio $(\mathrm{OR}=3.10, p=0.09)$ (Fig. 3b)

\section{Genes differentially expressed in MDD are enriched in aDMRs}

Given the absence of robust genetic associations with MDD, we also assessed for aDMR enrichment among 


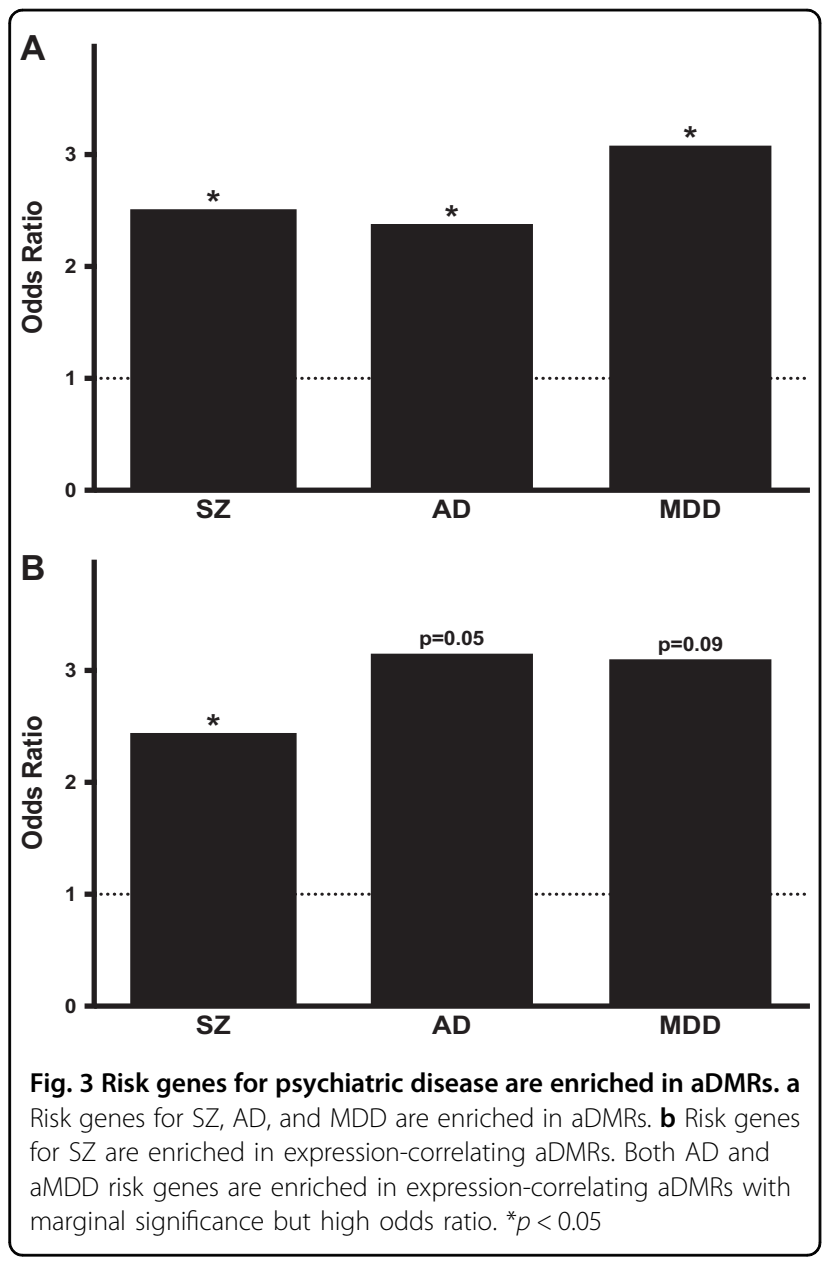

differentially expressed genes in MDD. We previously used meta-analysis to identify 566 genes for which expression consistently differed between MDD and control subjects ${ }^{31}$. DNAm data were available for analysis for 521 of these genes (Supplemental Table 11). These genes were enriched in aDMRs $(\mathrm{OR}=1.48, p=0.00012)$ and expression-correlating aDMRs $(\mathrm{OR}=1.91, \quad p=3.5 \times$ $10^{-5}$ ) (Supplemental Fig. 2).

Major findings from our primary dataset are replicated in a independent dataset and confirmed by meta-analysis

We next sought to replicate our findings in an independent dataset (See methods). DNAm sites for which data existed in the replication dataset were grouped into 267,370 CRs, and 4074 were differentially methylated between age groups, i.e., aDMRs. We found that top AGE genes were enriched in aDMRs $(\mathrm{OR}=2.19, p=9.24 \times$ $\left.10^{-19}\right)$. SZ risk genes, including the subset of proteincoding risk genes, were enriched in aDMRs $(\mathrm{OR}=2.01, p$ $=0.01$ and $\mathrm{OR}=3.88, p=0.00017$, respectively). $\mathrm{AD}$ risk genes were not enriched in aDMRs (OR $=1.37, p=0.37)$. MDD risk genes and genes differentially expressed in MDD were enriched in aDMRs $(\mathrm{OR}=3.32, p=0.02$ and $\mathrm{OR}=2.01, p=1.3 \times 10^{-9}$, respectively) (Table 2 ).

In a meta-analysis of the primary and replication datasets, we found that aDMRs were enriched among top AGE genes $\left(\mathrm{OR}=2.27, p=9.24 \times 10^{-23}\right)$; genetic risk genes for $\mathrm{SZ}(\mathrm{OR}=2.68, p=0.00012), \mathrm{AD}(\mathrm{OR}=2.66, p$ $=0.026)$, and $\operatorname{MDD}(\mathrm{OR}=4.11, p=0.0042)$; as well as genes differentially expressed in $\mathrm{MDD}(\mathrm{OR}=1.83, p=$ $2.1 \times 10^{-8}$ ).

Table 2 aDMR enrichment of gene sets for aging and psychiatric disease risk in two datasets and meta-analysis

\begin{tabular}{|c|c|c|c|}
\hline Gene Sets & Primary dataset & Replication dataset & Meta-analysis \\
\hline \multicolumn{4}{|l|}{ Age } \\
\hline All aDMRs & $O R=2.30, p=1.69 \times 10^{-27}$ & $O R=2.19, p=9.24 \times 10^{-19}$ & $O R=2.27, p=2.52 \times 10^{-23}$ \\
\hline Hypomethylated aDMRs only & $O R=2.08, p=3.85 \times 10^{-11}$ & $O R=1.88, p=5.55 \times 10^{-7}$ & $O R=2.28, p=2.04 \times 10^{-16}$ \\
\hline Hypermethylated aDMRs only & $O R=2.10, p=7.21 \times 10^{-18}$ & $O R=2.21, p=2.20 \times 10^{-13}$ & $O R=2.07, p=2.02 \times 10^{-11}$ \\
\hline \multicolumn{4}{|l|}{$S Z$} \\
\hline SZ risk genes & $O R=2.51, p=1.50 \times 10^{-4}$ & $O R=2.01, p=0.01$ & $O R=2.68, p=0.00012$ \\
\hline SZ risk genes (protein-encoding subset) & $O R=5.19, p=4.7 \times 10^{-7}$ & $O R=3.88, p=1.7 \times 10^{-4}$ & $O R=6.28, p=3.6 \times 10^{-8}$ \\
\hline \multicolumn{4}{|l|}{$A D$} \\
\hline $\mathrm{AD}$ risk genes & $O R=2.38, p=0.04$ & $\mathrm{OR}=\mathbf{1 . 3 7}, \boldsymbol{p}=0.37$ & $O R=2.66, p=0.0 .026$ \\
\hline \multicolumn{4}{|l|}{ MDD } \\
\hline MDD risk genes & $O R=3.15, p=0.05$ & $O R=3.32, p=0.02$ & $O R=4.11, p=0.004$ \\
\hline Expression-based MDD-associated genes & $O R=1.48, p=1.2 \times 10^{-4}$ & $O R=2.01, p=1.3 \times 10^{-9}$ & $O R=1.83, p=2.1 \times 10^{-8}$ \\
\hline
\end{tabular}

$D M R s$ differentially methylated regions, $O R$ odds ratio, $S Z$ schizophrenia, $A D$ Alzheimer's disease, MDD major depressive disorder Values in bold italics are statistically significant $(p<0.05)$ 


\section{Discussion}

In this study, we first characterized DNA methylation (DNAm) differences between younger and older adult subjects in the orbital frontal cortex (OFC). We found that age-associated DNAm changes are common, robust, bidirectional, concentrated in $\mathrm{CpG}$ island shelves and sea, and depleted in $\mathrm{CpG}$ islands. Next, we tested the hypothesis that age-associated DNAm changes contribute to age-associated gene expression changes and, by extension, susceptibility to psychiatric diseases. Supporting this hypothesis, we found genes that undergo ageassociated expression changes as well as genetic risk genes for three psychiatric diseases-SZ, AD, MDD-and genes differentially expressed in MDD are enriched in aDMRs. Further, we replicated the major findings in a large publically-available DNAm dataset, and confirmed them by meta-analysis of the primary and replication datasets. Together, our results provide evidence for DNAm as a mechanism for age-associated gene expression changes in the brain and support a role for DNAm in age-by-disease interactions through preferential targeting of diseaserelated genes.

\section{Characterization of age-associated DNAm changes in the human OFC}

Our findings with regard to age-associated DNAm changes in the OFC are largely consistent with previous studies of DNAm changes in normal aging human brains using Illumina DNAm arrays ${ }^{32-36}$. We found that the percentage of sites that underwent age-associated changes in DNAm was $3.9 \%(12,427 / 317,349)$, somewhat higher than reported in comparable studies ${ }^{32-38}$. For example, the largest comparable study found that only $\sim 1 \%$ of sites assessed exhibited DNAm changes ${ }^{32}$. The fact that all subjects in that study were 66 years of age or older may explain this difference. The rate of DNAm change slows with increasing age ${ }^{35}$, and, paradoxically, brain DNAm signatures may become more similar among individuals after the age of $75^{39}$. The greater percentage of ageassociated DNAm changes in our study may also be explained, in part, by the fact that we selected subjects to maximize group differences in two molecular markers of brain aging (BDNF and SST mRNA levels) ${ }^{12,13}$. Supporting this latter explanation is the observation that only $2.2 \%(7083 / 317,349)$ of sites, are differentially methylated in the replication dataset (from a study in which subjects were not selected using aging biomarkers).

Consistent with the observations of others ${ }^{40}$, we found that age-related DNAm changes were more likely to occur outside of CpG islands, specifically, in CpG island shelves and sea. This observation may reflect the presence of mechanisms that protect $\mathrm{CpG}$ islands from de novo methylation $^{41}$. Several studies of age-related DNAm changes using Illumina DNAm arrays report finding a greater percentage of changes occur in CpG islands ${ }^{32,33,36}$. Such reports, while accurate, do not correct for the fact that the probes on Illumina DNAm arrays are biased towards CpG islands.

Our observation that age-associated DNAm hypermethylation was much more common than hypomethylation is consistent with prior reports ${ }^{6}$. This finding, however, likely reflects the fact that the sites probed by Illumina DNAm arrays are biased toward CpG islands, promoters, and genic regions. In one study of peripheral blood that used whole-genome bisulphite sequencing instead of Illumina DNAm arrays, hypermethylation represented only $13 \%$ of the age-associated DNAm changes $^{42}$. In fact, most data suggest that the genome becomes globally hypomethylated with age and that the bulk of the DNAm loss occurs at the repetitive sequences in between genes ${ }^{6}$. The pattern of genic hypermethylation and intergenic hypomethylation with age is hinted at in our study by the observation that the likelihood of a site becoming hypermethylated with age appears to be a function of proximity to a $\mathrm{CpG}$ island.

\section{Age-associated changes in gene expression and DNAm}

Consistent with our hypothesis, both aDMRs and expression-correlating aDMRs are overrepresented among genes that undergo age-associated changes in expression. These findings suggest that age-associated changes in DNAm may be a mechanism contributing to age-associated expression changes for many genes across the genome. We previously demonstrated that DNAm at sites in a smaller number of genes implicated in psychiatric disease (several BDNF-related and GABA-related genes) correlated strongly with local gene expression ${ }^{16}$.

Pathway and gene ontology analysis of genes at which age-associated expression changes and expressioncorrelating DMRs converge identified some pathways and gene ontologies previously implicated in brain aging. For example, multiple canonical pathways and gene ontologies related to calcium signaling were identified by these analyses, and calcium signaling is already appreciated as one of the most prominent cellular and molecular functions to be dysregulated in brain aging ${ }^{43-46}$. Indeed, calcium signaling proteins are thought to be promising targets for drugs to treat age-related brain diseases ${ }^{46}$ and a transgenic mouse that overexpresses the L-type calcium channel $\mathrm{Ca}_{\mathrm{v}} 1.3$ in a forebrain-specific manner has been advanced as an animal model of normal brain aging ${ }^{47}$. However, these analyses also identified some pathways and gene ontologies not typically associated with brain aging such as opioid signaling, a potentially promising toper for future investigation high burden of chronic pain in older adults ${ }^{48-50}$. Pathway and gene ontology analysis using only genes to which expression-correlating aDMRs were annotated identified 
some distinct canonical pathways and gene ontologies from the analyses that also incorporated information about genes that undergo age-associated gene expression. Most notably, this gene set was distinctly enriched in canonical pathways and gene ontologies associated with brain pathology including gliomas and other malignancies as well as neuropathic pain thus suggesting that expression of these genes may only be altered in specific contexts like particular brain pathologies, etc. However, because Illumina DNAm arrays are biased towards particular regions of the genome, results derived from pathway analysis software created for gene expression arrays should be interpreted with caution ${ }^{51}$.

Age-associated DNAm changes and psychiatric risk genes

Consistent with our prediction, genes associated with psychiatric diseases through genetic studies were enriched in aDMRs. However, the specific sets of risk genes affected were different than predicted. Contrary to our prediction, SZ risk genes were the psychiatric diseaseassociated gene set that was most strongly enriched in aDMRs (greater OR and lower $p$-value), whereas aDMR enrichment for AD risk genes were the most weakly enriched (lower OR and higher $p$-value). MDD risk genes defined based on genetic studies as well as expressionbased MDD-associated genes were enriched in ageassociated aDMRs and thus consistent with our prediction,

Our findings that psychiatric disease risk genes are also enriched in expression-correlating aDMRs suggest that DNAm changes in these genes often alter their expression. Together, these observations support a role for ageassociated DNAm changes in age-by-disease interactions. Our Age-by-Disease Model posits that these age-associated changes in gene expression then push biological processes in a direction that promotes a physiological state in the brain that is more susceptible to psychiatric diseases like SZ, MDD, and AD when occurring out of their chronological context, i.e., earlier in life $^{2-4}$. Additional pushes by genetic or environmental factors may then be sufficient to generate brain dysfunction that gives rise to psychiatric diseases.

We predicted that any age-associated DNAm changes relevant to SZ etiology and/or pathophysiology would occur during neurodevelopment, be complete by late adolescence, and not be detectable because our age groups did not span the neurodevelopmental period. Our observation that SZ risk genes are enriched in aDMRs instead suggests that age-associated DNAm changes relevant to SZ may occur during neurodevelopment, but also continue as the brain ages into and through adulthood. Indeed, many important developmental genes are associated with DNAm changes during both early development and adulthood ${ }^{52,53}$ The age-associated
DNAm changes in SZ risk genes during adulthood may impact the longitudinal course of SZ and suggests a potential mechanism for some of the changes observed in subjects with SZ across adulthood including cortical gray matter thinning ${ }^{54,55}$ and progressive cognitive decline ${ }^{56,57}$.

We predicted that AD risk genes would be enriched in aDMRs because the age groups studied captured the extremes of the transition from younger to older adult, a period during which one might expect AD-related biological processes to be particularly active. The AD risk gene set was enriched in aDMRs, and the potential importance of this observation to AD etiology is supported by recent studies showing that altered DNAm in AD susceptibility genes is associated with $A D$ neuropathology ${ }^{58,59}$. Notably, these studies found cortexspecific ANK1 hypermethylation to be one of the alterations most strongly associated with AD neuropathology and we found $A N K 1$ to contain multiple large relatively hypermethylated aDMRs in this study. The fact that $\mathrm{AD}$ risk gene set was the psychiatric disease-associated gene set that was most weakly enriched in aDMRs may be explained by the power lost but studying a limited number of genes ( $N=25$ after data filtering). Also, the fact that the definition of 'risk gene' differed among SZ, MDD, and $\mathrm{AD}$ may have caused divergence from our predictions. Risk genes for SZ and most of the AD risk genes were defined based on their proximity to GWAS-associated common genetic variants for each disease. The genes associated with the rare deterministic genetic mutations for $\mathrm{AD}$ were also considered $\mathrm{AD}$ risk genes. Because so few common genetic variants have robustly been associated with MDD, risk genes for MDD were defined based on proximity to common genetic variants found to be associated with MDD at the level of $p<10^{-5}$ in a single large study and thus quite different from the robust associations demonstrated for SZ and AD. The role in disease etiology and pathophysiology of risk genes defined in these various ways are likely different, as is the way they would likely interact with DNAm changes. Because MDD has a bimodal age of onset ${ }^{60,61}$ and can first present or recur at any adult age, we predicted that MDD risk genes would be enriched in aDMRs to a degree intermediate between $\mathrm{SZ}$ and $\mathrm{AD}$ given that our age groups may not be particularly well-suited for detecting age-associated DNAm changes relevant to MDD that is limited to early or late adulthood. Our observation that MDD risk genes, and genes differentially expressed in the brains of MDD subjects, are enriched in aDMRs is consistent with this prediction. The accumulation of age-associated DNAm changes over time in genes associated with MDD may help explain the high rates of depressive symptoms among individuals in late life $^{62,63}$, as we have previously hypothesized $^{2,3}$. 


\section{Conclusion}

This study provides further evidence for DNAm as a mechanism contributing to age-associated gene expression changes. Additionally, it provides further support for age-associated DNAm changes as a mechanism for mediating the age-by-disease changes in gene expression previously hypothesized to contribute to the etiology and pathophysiology of psychiatric diseases.

\section{Acknowledgements}

This work was supported by NIH-NIMH Grants T32 MH016804 (BCM), KL2 TR001856 (BCM), K23 MH112798 (BCM), and R01 MH093723 (ES), and by a National Alliance for Research on Schizophrenia and Depression (NARSAD) award (ES). We thank Dr. Leon French for feedback on the analysis and manuscript. David A. Lewis currently receives investigator-initiated research support from Pfizer and in 2017 served as a consultant to Merck.

\section{Author details}

${ }^{1}$ Department of Psychiatry, University of Pittsburgh, Pittsburgh, PA, USA. ${ }^{2}$ Department of Biostatistics, University of Pittsburgh, Pittsburgh, PA, USA ${ }^{3}$ Campbell Family Mental Health Research Institute of CAMH, Departments of Psychiatry, and of Pharmacology and Toxicology, University of Toronto, Toronto, ON, Canada

\section{Conflict of interest}

The authors declare that they have no conflict of interest.

\section{Publisher's note}

Springer Nature remains neutral with regard to jurisdictional claims in published maps and institutional affiliations.

Supplementary information accompanies this paper at (https://doi.org/ 10.1038/s41398-019-0372-2).

Received: 8 February 2018 Revised: 2 December 2018 Accepted: 9 December 2018

Published online: 29 January 2019

\section{References}

1. The Global Burden of Disease: 2004 Update. www.who.int/healthinfo/ global_burden_disease/GBD report_2004update full.pdf. World Health Organization (2004)

2. McKinney, B. C., Oh, H. \& Sibille, E. Age-by-disease biological interactions: implications for late-life depression. Front. Genet. 3, 237 (2012).

3. McKinney, B. C. \& Sibille, E. The age-by-disease interaction hypothesis of latelife depression. Am. J. Geriatr. Psychiatry 21, 418-432 (2013).

4. Sibille, E. Molecular aging of the brain, neuroplasticity, and vulnerability to depression and other brain-related disorders. Dialog. Clin. Neurosci. 15, 53-65 (2013).

5. Fraga, M. F. \& Esteller, M. Epigenetics and aging: the targets and the marks. Trends Genet. 23, 413-418 (2007).

6. Jones, M. J., Goodman, S. J. \& Kobor, M. S. DNA methylation and healthy human aging. Aging Cell 14, 924-932 (2015).

7. Talens, R. P. et al. Epigenetic variation during the adult lifespan: cross-sectiona and longitudinal data on monozygotic twin pairs. Aging Cell 11, 694-703 (2012).

8. Fraga, M. F. et al. Epigenetic differences arise during the lifetime of monozygotic twins. Proc. Natl Acad. Sci. USA 102, 10604-10609 (2005).

9. van Dongen, J. et al. Epigenetic variation in monozygotic twins: a genomewide analysis of DNA methylation in buccal cells. Genes 5, (347-365 (2014).

10. Baubec, T. \& Schubeler, D. Genomic patterns and context specific interpretation of DNA methylation. Curr. Opin. Genet. Dev. 25, 85-92 (2014).

11. Lu, T. et al. Gene regulation and DNA damage in the ageing human brain. Nature 429, 883-891 (2004).
12. Glorioso, C. \& Sibille, E. Between destiny and disease: genetics and molecular pathways of human central nervous system aging. Prog. Neurobiol. 93, 165-181 (2011)

13. Erraji-Benchekroun, L. et al. Molecular aging in human prefrontal cortex is selective and continuous throughout adult life. Biol. Psychiatry 57, 549-558 (2005).

14. Seney, M. L. et al. The role of genetic sex in affect regulation and expression of GABA-related genes across species. Front. Psychiatry 4, 104 (2013).

15. Adkins, R. M., Krushkal, J., Tylavsky, F. A. \& Thomas, F. Racial differences in genespecific DNA methylation levels are present at birth. Birth. Defects Res. A 91 728-736 (2011).

16. McKinney, B. C. et al. Hypermethylation of BDNF and SST genes in the orbital frontal cortex of older individuals: a putative mechanism for declining gene expression with age. Neuropsychopharmacology 40, 2604-2613 (2015).

17. Mozhui, K., Smith, A. K. \& Tylavsky, F. A. Ancestry dependent DNA methylation and influence of maternal nutrition. PLoS One 10, e0118466 (2015).

18. Terry, M. B. et al. Genomic DNA methylation among women in a multiethnic New York City birth cohort. Cancer Epidemiol. Biomark. Prev. 17, 2306-2310 (2008)

19. Nestler, E. J., Pena, C. J., Kundakovic, M., Mitchell, A. \& Akbarian, S. Epigenetic basis of mental illness. Neuroscientist 22, 447-63 (2015).

20. Price, M. E. et al. Additional annotation enhances potential for biologicallyrelevant analysis of the Illumina Infinium HumanMethylation450 BeadChip array. Epigenetics Chromatin 6, 4 (2013).

21. Guintivano, J., Aryee, M. J. \& Kaminsky, Z. A. A cell epigenotype specific mode for the correction of brain cellular heterogeneity bias and its application to age, brain region and major depression. Epigenetics 8, 290-302 (2013).

22. French, L., Ma, T., Oh, H., Tseng, G. C. \& Sibille, E. Age-related gene expression in the frontal cortex suggests synaptic function changes in specific inhibitory neuron subtypes. Front. Aging Neurosci. 9, 162 (2017).

23. Jaffe, A. E. et al. Mapping DNA methylation across development, genotype and schizophrenia in the human frontal cortex. Nat. Neurosci. 19, 40-47 (2016).

24. Gardiner-Garden, M. \& Frommer, M. CpG islands in vertebrate genomes. J. Mol. Biol. 196, 261-282 (1987).

25. Irizarry, R. A. et al. The human colon cancer methylome shows similar hypoand hypermethylation at conserved tissue-specific CpG island shores. Nat Genet. 41, 178-186 (2009).

26. Schizophrenia Working Group of the Psychiatric Genomics C. Biological insights from 108 schizophrenia-associated genetic loci. Nature 511, 421-427 (2014).

27. Lambert, J. C. et al. Meta-analysis of 74,046 individuals identifies 11 new susceptibility loci for Alzheimer's disease. Nat. Genet. 45, 1452-1458 (2013).

28. Colonna, M. \& Wang, Y. TREM2 variants: new keys to decipher Alzheimer disease pathogenesis. Nat. Rev. Neurosci. 17, 201-207 (2016).

29. Van Cauwenberghe, C., Van Broeckhoven, C. \& Sleegers, K. The genetic landscape of Alzheimer disease: clinical implications and perspectives. Genet. Med. 18, 421-30 (2015).

30. consortium, C. Sparse whole-genome sequencing identifies two loci for major depressive disorder. Nature 523, 588-591 (2015).

31. Ding, $Y$. et al. Molecular and genetic characterization of depression: overlap with other psychiatric disorders and aging. Mol. Neuropsychiatry 1, 1-12 (2015).

32. Yang, J. et al. Association of DNA methylation in the brain with age in older persons is confounded by common neuropathologies. Int. J. Biochem. Cell. Biol. 67, 58-64 (2015).

33. Hernandez, D. G. et al. Distinct DNA methylation changes highly correlated with chronological age in the human brain. Hum. Mol. Genet. 20, 1164-1172 (2011).

34. Horvath, S. DNA methylation age of human tissues and cell types. Genome Biol. 14, R115 (2013).

35. Numata, S. et al. DNA methylation signatures in development and aging of the human prefrontal cortex. Am. J. Hum. Genet. 90, 260-272 (2012).

36. Day, $\mathrm{K}$. et al. Differential DNA methylation with age displays both common and dynamic features across human tissues that are influenced by CpG landscape. Genome Biol. 14, R102 (2013).

37. Horvath, $\mathrm{S}$. et al. Aging effects on DNA methylation modules in human brain and blood tissue. Genome Biol. 13, R97 (2012).

38. Gibbs, J. R. et al. Abundant quantitative trait loci exist for DNA methylation and gene expression in human brain. PLoS Genet. 6, e1000952 (2010).

39. Oh, G. et al. Epigenetic assimilation in the aging human brain. Genome Biol. 17 76 (2016). 
40. Numata, S., Ye, T., Herman, M. \& Lipska, B. K. DNA methylation changes in the postmortem dorsolateral prefrontal cortex of patients with schizophrenia. Front. Genet. 5, 280 (2014).

41. Brandeis, M. et al. Sp1 elements protect a CpG island from de novo methylation. Nature 371, 435-438 (1994).

42. Heyn, H. et al. Distinct DNA methylomes of newborns and centenarians. Proc Natl Acad. Sci. USA 109, 10522-10527 (2012).

43. Hands, S., Sinadinos, C. \& Wyttenbach, A. Polyglutamine gene function and dysfunction in the ageing brain. Biochim. Biophys. Acta 1779, 507-521 (2008).

44. Cheng, X., Wu, J., Geng, M. \& Xiong, J. Role of synaptic activity in the regulation of amyloid beta levels in Alzheimer's disease. Neurobiol. Aging 35, 1217-1232 (2014).

45. Yu, X. W., Oh, M. M. \& Disterhoft, J. F. CREB cellular excitability, and cognition: implications for aging. Behav. Brain Res. 322, 206-211 (2016).

46. Chandran, R. et al. Cellular calcium signaling in the aging brain. J. Chem. Neuroanat. 95, 95-114 (2019).

47. Krueger, J. N. et al. A novel mouse model of the aged brain: over-expression of the L-type voltage-gated calcium channel CaV1.3. Behav. Brain Res. 322(Pt B), 241-249 (2017).

48. Blyth, F. M. \& Noguchi, N. Chronic musculoskeletal pain and its impact on older people. Best Pract. Res. Clin. Rheumatol. 31, 160-168 (2017).

49. Rice, A. S., Smith, B. H. \& Blyth, F. M. Pain and the global burden of disease. Pain 157, 791-796 (2016).

50. Schaefer, C. et al. Burden of illness associated with peripheral and central neuropathic pain among adults seeking treatment in the United States: a patient-centered evaluation. Pain Med. 15, 2105-2119 (2014).

51. Harper, K. N., Peters, B. A. \& Gamble, M. V. Batch effects and pathway analysis: two potential perils in cancer studies involving DNA methylation array analysis. Cancer Epidemiol. Biomark. Prev. 22, 1052-1060 (2013).
52. Rakyan, V. K. et al. Human aging-associated DNA hypermethylation occurs preferentially at bivalent chromatin domains. Genome Res. 20, 434-439 (2010).

53. Teschendorff, A. E. et al. Age-dependent DNA methylation of genes that are suppressed in stem cells is a hallmark of cancer. Genome Res. 20, 440-446 (2010).

54. van Haren, N. E. et al. Changes in cortical thickness during the course of illness in schizophrenia. Arch. Gen. Psychiatry 68, 871-880 (2011).

55. van Haren, N. E. et al. Progressive brain volume loss in schizophrenia over the course of the illness: evidence of maturational abnormalities in early adulthood. Biol. Psychiatry 63, 106-113 (2008).

56. Irani, F., Kalkstein, S., Moberg, E. A. \& Moberg, P. J. Neuropsychological performance in older patients with schizophrenia: a meta-analysis of crosssectional and longitudinal studies. Schizophr. Bull. 37, 1318-1326 (2011).

57. Thompson, W. K. et al. Characterizing trajectories of cognitive functioning in older adults with schizophrenia: does method matter? Schizophr. Res. 143, 90-96 (2013).

58. De Jager, P. L. et al. Alzheimer's disease: early alterations in brain DNA methylation at ANK1, BIN1, RHBDF2 and other loci. Nat. Neurosci. 17, 1156-1163 (2014).

59. Lunnon, K. et al. Methylomic profiling implicates cortical deregulation of ANK1 in Alzheimer's disease. Nat. Neurosci. 17, 1164-1170 (2014).

60. Weissman, M. M Handbook of Anxiety Disorders. (Elsevier Science Publishers, New York, 1987).

61. Rohrbaugh, R. M., Sholomskas, D. E. \& Giller, E. L. Jr. Lifetime course of chronic depression in older men. J. Geriatr. Psychiatry Neurol. 2, 89-95 (1989).

62. Koenig, H. G. \& Blazer, D. G. Epidemiology of geriatric affective disorders. Clin. Geriatr. Med. 8, 235-251 (1992).

63. Weissman, M. M. et al. Affective disorders in five United States communities. Psychol. Med. 18, 141-153 (1988). 